\title{
A Randomized Controlled Trial with a Medical Device Containing Sodium Hyaluronate and Nicotinic Acid to Increase the Efficacy of Ultraviolet Phototherapy in Psoriasis
}

Balázs Bende · Róbert Kui · Annamária Németh · Mariann Borsos •

Zoltán Tóbiás · Gábor Erős (D) · Lajos Kemény · Eszter Baltás

Received: April 1, 2020 / Published online: May 9, 2020

(C) The Author(s) 2020

\section{ABSTRACT}

Introduction: The dry and scaly skin of psoriatic patients decreases the efficacy of ultraviolet B (UVB) phototherapy. Different agents are used to facilitate the transmission of light, but most of these preparations are cosmetically unfavorable. We have tested a novel preparation containing sodium hyaluronate and nicotinic acid (UV Fotogel®; Pernix Ltd.) with the double aim

Digital Features To view digital features for this article go to https://doi.org/10.6084/m9.figshare.12162021.

B. Bende · R. Kui · G. Erős $(\bowtie) \cdot$ L. Kemény ·

E. Baltás

Department of Dermatology and Allergology,

University of Szeged, Szeged, Hungary

e-mail: eros.gabor@med.u-szeged.hu

A. Németh

Clinical Research Coordination Centre, University

of Szeged, Szeged, Hungary

M. Borsos

Adware Research Ltd., Balatonfüred, Hungary

Z. Tóbiás

Department of Otorhinolaryngology and Head and

Neck Surgery, University of Szeged, Szeged, Hungary

L. Kemény

MTA SZTE Dermatological Research Group, Szeged, Hungary

L. Kemény

HCEMM SZTE Skin Research Group, Szeged, Hungary to improve the efficacy of UVB phototherapy and assess the cosmetic acceptability of the preparation.

Methods: Ninety patients with plaque psoriasis were enrolled in the study, of whom 44 received narrow-band UVB (NB-UVB) phototherapy. Prior to phototherapy, one side of the patient's body was treated with UV Fotogel while the other side served as a control. The other 46 patients used the preparation at their homes before regular sunbathing. The Local Psoriasis Severity Index (L-PSI), cosmetic acceptability and tolerability were recorded. The median values with the 25 th and 75 th percentiles $(25 p$ and $75 p$, respectively) were determined for the UV Fotogel-treated and control sites and then compared.

Results: The sides of the body to which UV Fotogel was applied prior to NB-UVB phototherapy had a significantly lower median L-PSI score than the non-treated control sides at the end of the treatment (1.0 [25p-75p: 0.0-2.0] vs. 2.0 [1.0-3.0], respectively). The application of UV Fotogel prior to sunbathing also led to a significant decrease in L-PSI score. There was a significant reduction in the median L-PSI score of patients at the final visit compared to baseline (2.5 [25p-75p: 1.5-3.5] vs. 6.0 [6.0-7.0], respectively). Use of the preparation was not accompanied by considerable adverse effects, and the patients found it cosmetically acceptable. Application of UV Fotogel prior to 
sunbathing was well tolerated by the patients, and the cosmetic acceptability was also good.

Conclusion: UV Fotogel is potentially a useful device for enhancement of the efficacy of phototherapy in patients with psoriasis.

Keywords: Optical cleaning; Phototherapy; Psoriasis; Ultraviolet B

\section{Key Summary Points}

Why carry out this study?

Phototherapy is a cornerstone in the treatment of psoriasis; however, dry and scaly skin decreases the efficacy of phototherapy.

Many treatments used to improve the efficacy of phototherapy have unfavorable cosmetic properties or absorb ultraviolet (UV) light, requiring higher UV doses to be effective.

The aim of this study was to test whether our novel medical device (UV Fotogel ${ }^{\circledR}$ ) enhances UV phototherapy in psoriatic patients.

What was learned from the study?

UV Fotogel considerably increased the efficacy of UV phototherapy.

UV Fotogel was well tolerated by the patients.

In contrast to compounds with similar effects, UV Fotogel is cosmetically acceptable.

\section{INTRODUCTION}

soriasis is a chronic autoimmune disorder, with a prevalence in adults that varies from $0.91 \%$ (USA) to $8.5 \%$ (Norway) [1], that considerably decreases the health-related quality of life. Although chronic plaque psoriasis, which is the most common type of psoriasis, can be diagnosed without major difficulties, the disease may still be underdiagnosed and undertreated [2-4]. Several treatment options are available for psoriasis. Topical therapy is typically chosen in mild to moderate manifestations, with corticosteroids [5], vitamin D3 analogues [6] and the combination thereof [7] shown to be effective. In more severe cases, systemic therapy is indicated, most commonly with immunosuppressants (e.g., methotrexate, cyclosporine), immune modulators (e.g., leflunomide), and biological agents [8-10]. In addition to these different pharmacological approaches, phototherapy is also considered to be a safe and effective method for the treatment of psoriasis. Many types of phototherapy use ultraviolet B (UVB) light [11], with the most effective wavelengths of the UVB spectrum being between 310 and $320 \mathrm{~nm}$. Wavelengths of 280-300 $\mathrm{nm}$ have a reduced therapeutic effect and cause severe and unnecessary skin inflammation [12-14]. The efficacy of phototherapy is influenced by the reflection of UV light from the skin surface, with dry, scaly skin reflecting more UV light than healthy skin; accordingly, a lower proportion of the radiation penetrates the psoriatic lesions, thereby decreasing the therapeutic effect. Thus, the efficacy of phototherapy might be improved by optimizing skin transmittance and irradiating at a wavelength of 310-320 nm. Different topicals have been tested with this goal (e.g., petrolatum, basis cream, glycerine, olive oil, aqueous cream, Eucerin skin products, Kamillosan ointment, paraffin ointment, mineral oil [15-17]); however, some preparations had unfavorable cosmetic properties or absorbed light in the UV spectrum, requiring higher UVB doses to be effective.

The aims of this study was to test a novel medical device containing polyethylene glycol 4000, nicotinic acid, and sodium hyaluronate (UV Fotogel ${ }^{\circledR}$ ) for the enhancement of phototherapy in psoriatic patients and to monitor its efficacy and tolerability. The investigation was performed in two parts. In Part I, the preparation was applied before the patient received narrow-band UVB therapy (NB-UVB), and in Part II, UV Fotogel was used by the patients prior to going sunbathing. 


\section{METHODS}

\section{UV Fotogel}

The gel comprises polyethylene glycol 4000, nicotinic acid, sodium hyaluronate, and purified water. It is classified as a CE-marked Class I medical device produced by Pernix Ltd. (Zalaegerszeg, Hungary).

\section{Patients}

Of the 90 patients enrolled in the study, 44 patients participated in Part I (24 males, 20 females; mean age 57.0 [range 26-82] years) and 46 participated in Part II (30 males and 16 females; mean age 57.2 [range 26-82] years). All patients had plaque psoriasis. The inclusion criteria were: minimum age of 18 years; ability and intention to follow the instructions of the investigator; communicated well with the study personnel; indication for NB-UVB; disintegration and desquamation of stratum corneum; a degree of desquamation of at least 2 points according to the Local Psoriasis Severity Index (L-PSI) score; and provided a signed informed consent form. The exclusion criteria were: application of any systemic and topical antipsoriatic drugs (e.g., methotrexate, acitretin, cyclosporine, biological therapies); use of potentially photosensitizing drugs; anamnesis of another disease accompanied by photosensitivity; and progression of psoriasis during previous NB-UVB or psoralen and UVA therapy.

The study was approved by the National Institute of Pharmacy and Nutrition (Hungary) (permission numbers: OGYÉI/723/2017 and OGYÉI/28552/2017) and was performed in accordance with the Helsinki Declaration of 1964 and its later amendments. Informed consent was obtained from all participants.

\section{Study Protocol}

The aim of Part I was to test the efficacy of UV Fotogel in patients receiving NB-UVB treatment (Fig. 1). In this part of the study, the patients were enrolled on day 0 (visit 1 ), at which time they signed the informed consent agreement

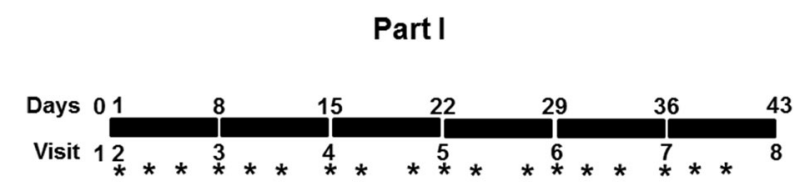

Part II

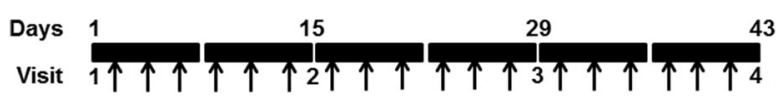

Fig. 1 Study protocol. Asterisks indicate the times of narrow-band ultraviolet B (NB-UVB) treatments (Part I), and the arrows indicate when the patient went sunbathing (Part II). Although the patients were instructed to sunbathe 3-5 times weekly, the actual number of times sunbathing per week differed for each patient. In Part I, the UV Fotogel was applied to parts of the body before NB-UVB therapy; in Part II, UV Fotogel applied by the patients prior to sunbathing

and the baseline L-PSI scores were recorded. In addition, the minimal erythema dose (MED) was determined for each patient (range at baseline $0.303-0.365 \mathrm{~J} / \mathrm{cm}^{2}$ ). On the next day (visit 2), the patients received their first phototherapy treatment. One to three ipsilateral plaques on the trunk and limbs and on both sides of the body were chosen for treatment. Randomization and application of UV Fotogel was performed by a nurse specialized in phototherapy. Prior to the NB-UVB treatment, UV Fotogel was applied to one side of the body only; the other side did not receive UV Fotogel. The dose of the first treatment was 0.75 MED. In subsequent treatments, the dose was increased in increments of $25 \%$. When considerable erythema was present, the last dose was not increased. NB-UVB was administered with a UV Therapy System UV 1002 (Herbert Waldmann GmbH \& Co., Villingen-Schwenningen, Germany). The cumulative total dose varied between 2.555 to $27.77 \mathrm{~J} / \mathrm{cm}^{2}$. NB-UVB treatments were given three times a week, and no other anti-psoriatic treatments were allowed throughout the study period. When desquamation ceased, the study was terminated for a given patient. The study was also terminated when patients achieved an L-PSI score of $<4$. 
The duration of treatment for all patients participating in Part 1 was a minimum of 3 weeks and not longer than 6 weeks.

The aim of Part II, which was conducted between 10 July 2017 and 20 September 2017, was to examine the tolerability of UV Fotogel applied prior to sunbathing at the patients' homes (Fig. 1). Two lesions were chosen for each patient, and the size and L-PSI score of each lesion were determined at baseline. The patients were provided the UV Fotogel to be applied and instructed to sunbathe for 15-30 min three to five times weekly between 8:30 am and 10:30 am or between 3:30 pm and 6:30 pm. Patients were instructed to apply UV Fotogel to the chosen lesions and to apply a sun protection preparation to the rest of the exposed skin prior to sun exposure. The length of time and other conditions of each sunbath were recorded. The patient attended clinic visits at 2-week intervals. The clinical study was terminated when cessation of the desquamation was observed. Enrollment in Part II of the study was a minimum of 4 weeks and not longer than 6 weeks.

\section{L-PSI, Cosmetic Acceptability, and Tolerability}

The L-PSI of each patient was evaluated by an experienced dermatologist. The L-PSI score was calculated by monitoring erythema, infiltration, and desquamation and then scoring each of these parameters with a score of 0 to 4 ( 0 : not present; 1 : mild, 2 : moderate; 3 : severe; 4 : very severe).

Cosmetic acceptability was evaluated on a 5-point scale (1: poor; 2: sufficient; 3: good; 4: very good; 5 : excellent).

Patients were asked whether they experienced any of the following symptoms: burning sensations, stabbing pain, itching, stretching, and tingling. These parameters of tolerability were recorded on a 4-point scale (1: not present; 2: mild; 3: moderate; 4 : severe).
Part I was a randomized and single-blind study (the evaluating dermatologist was blinded to the treatment sides), whereas Part II was an open study.

\section{Statistical Analysis}

Statistical analysis was performed with the SigmaStat statistical software package for Windows (Jandel Scientific, Erkrath, Germany). The Shapiro-Wilk test was used to check normality. The values obtained varied significantly from the pattern expected for a population with normal distribution. Hence, non-parametric methods were chosen for the comparison. The Wilcoxon signed-rank test was applied to compare data obtained before and after treatments, and the Mann-Whitney rank sum test was used to analyze the differences between control and treated sides. Median values are presented with the 25 th and 75 th percentiles (25p and 75p, respectively). Statistical significance was set at $p<0.05$.

\section{RESULTS}

In Part I, one patient left the study due to progression of the disease (systemic therapy was required). Cessation of desquamation was observed in three patients by the fourth visit. The study was terminated for an additional nine patients at visit 4,18 patients at visit 5 , and eight patients at visit 6 as these patients had L-PSI scores $<4$. With the exception of one patient, the improvement leading to termination of the study was detected on the UV Fotogel-treated side (Fig. 2).

In Part II, one patient decided to terminate his enrollment after visit 2 . Another patient quit due to an adverse effect of the treatment (burning sensation) but attended the final visit. Desquamation had ceased in 40 patients by visit 3 and in the remaining four patients by visit 4 (Fig. 2). 


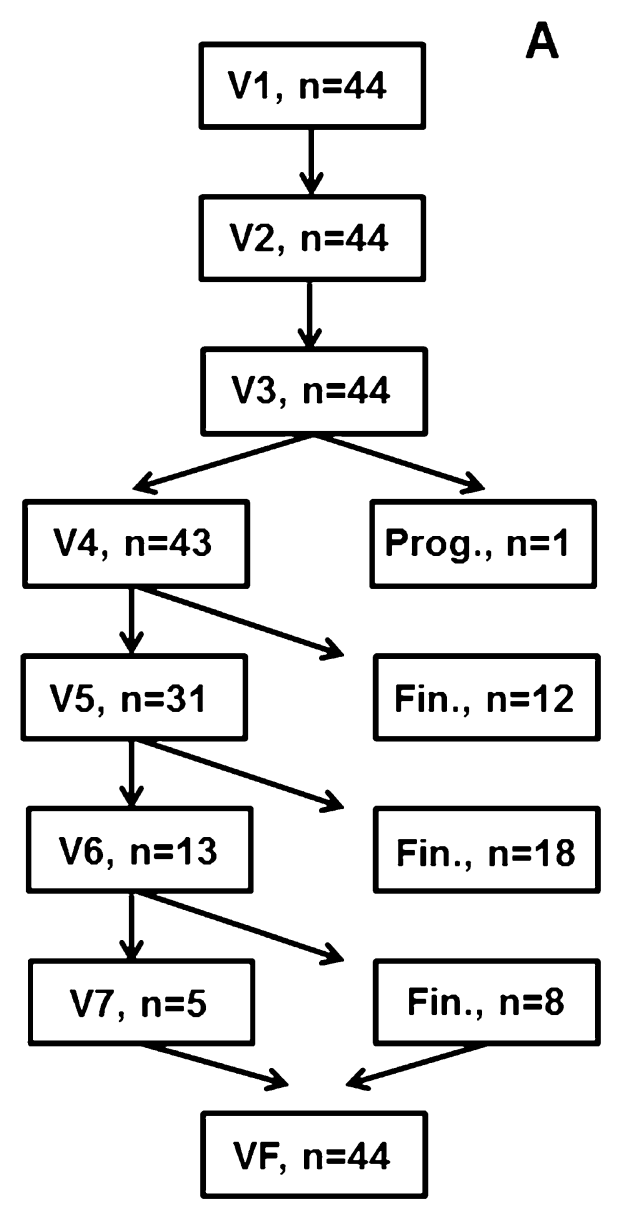

Fig. 2 Flow chart of study progression and patient distribution. a Part I, b Part II. $V 1, V 2 \ldots V 7$ visit 1 , visit 2 , and all subsequent visits up to visit 7 , Prog. progression of the disease, Fin. number of patients finishing the study

\section{At the Final Visit, Application of UV Fotogel Prior to NB-UVB Had Led to Significantly Lower Scores for L-PSI, Erythema, Infiltration, and Desquamation on Treated Sides Compared to the Sides Not Treated with UV Fotogel}

The median L-PSI score for both sides at baseline was 7.0 (25p-75p: 6.0-8.75). Compared to the baseline score, there was a statistically significant amelioration in median L-PSI scores on both the control (NB-UVB only) and treated (NB-UVB plus UV Fotogel) sides at the final visit, but the median L-PSI score of the treated side was significantly lower than that of the

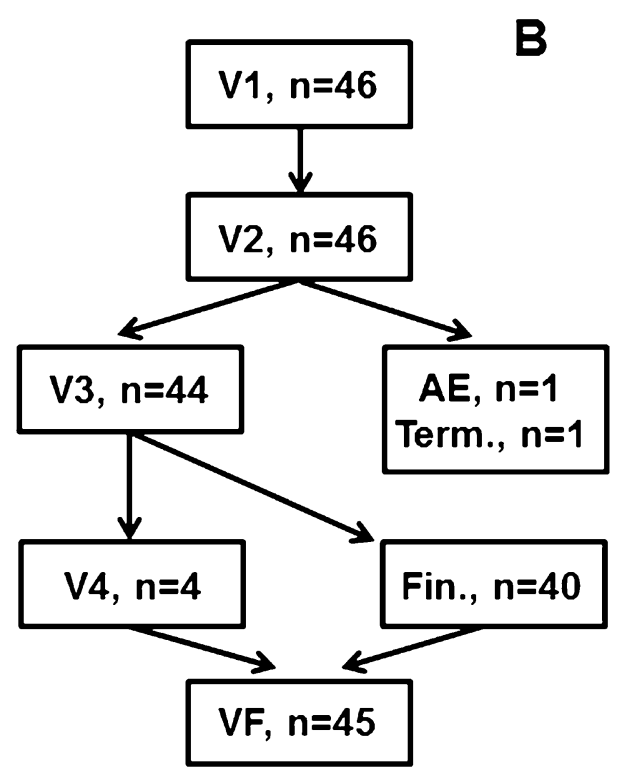

at the given time due to cessation of desquamation or Local Psoriasis Severity Index (L-PSI) score $<4, A E$ adverse event, $V F$ final visit, Term Termination of the study due to own decision

control side (1.0 [25p-75p: 0.0-2.0] vs. 2.0 [1.0-3.0], respectively) (Fig. 3a).

The median erythema score at baseline was 2.0 (25p-75p: 2.0-3.0). Compared to the median baseline score, there was a statistically significant reduction in this score on both the control and treated sides at the final visit, but the median erythema score of the treated side was significantly lower than that of the control side, indicating a greater decrease in erythema (1.0 [25p-75p: $0.0-1.0]$ vs. 1.0 [1.0-1.0], respectively) (Fig. 3b).

The median infiltration value after treatment at visit 1 was 2.0 (25p-75p: $2.0-3.0)$. The median infiltration score at subsequent visits decreased on both the treated and control sides, 

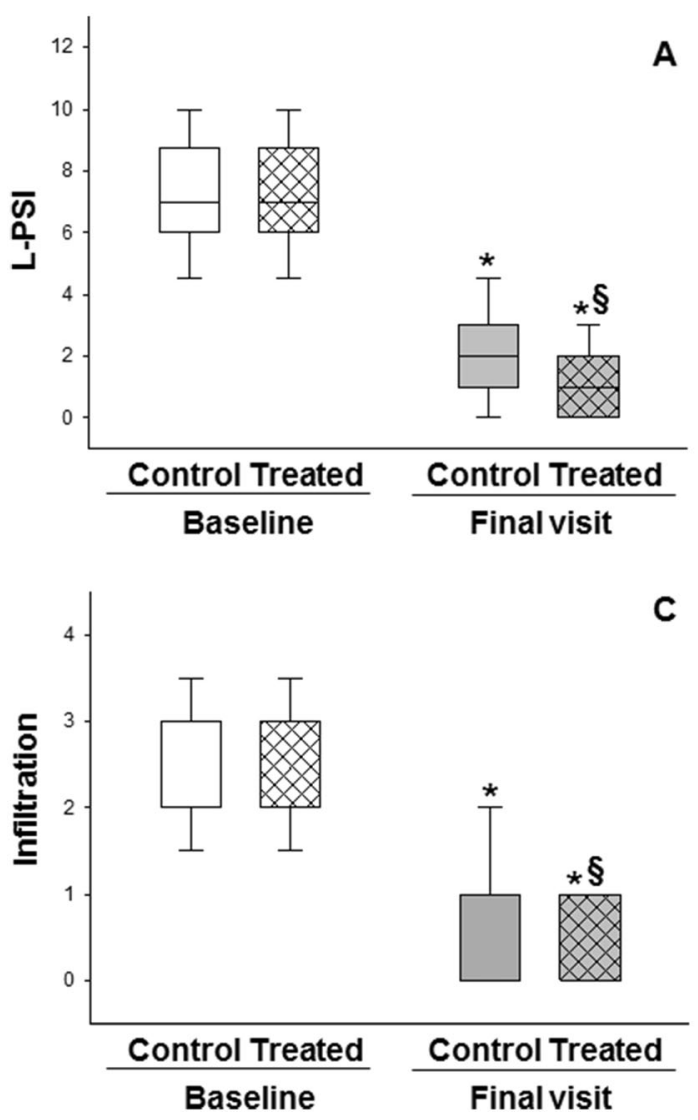

Fig. 3 Box plot of changes in the severity of psoriasis from baseline to the final visit. Median and 25th and 75th percentiles are shown. Asterisk indicates a significant difference $\left({ }^{*} p<0.05\right)$ between values at baseline and those at the final visit; the double $S$ symbol indicates a significant difference $\left({ }^{\$} p<0.05\right)$ between the treated side and the control side. a Changes in the L-PSI scores of

however improvement of the skin, as indicated by the median infiltration score, on the side treated with UV Fotogel surpassed that of the control side at the final visit $(0.0$ [25p-75p: 0.0-1.0] vs. 1.0 [0.0-1.0], respectively) (Fig. 3c).

The median desquamation score at baseline was 2.0 (25p-75p: 2.0-3.0). Compared to the median baseline score, there was a statistically significant reduction in this score on both the control and treated sides at the final visit, but the reduction, as indicated by the mean desquamation score, was significantly greater on the side treated with UV Fotogel than on the
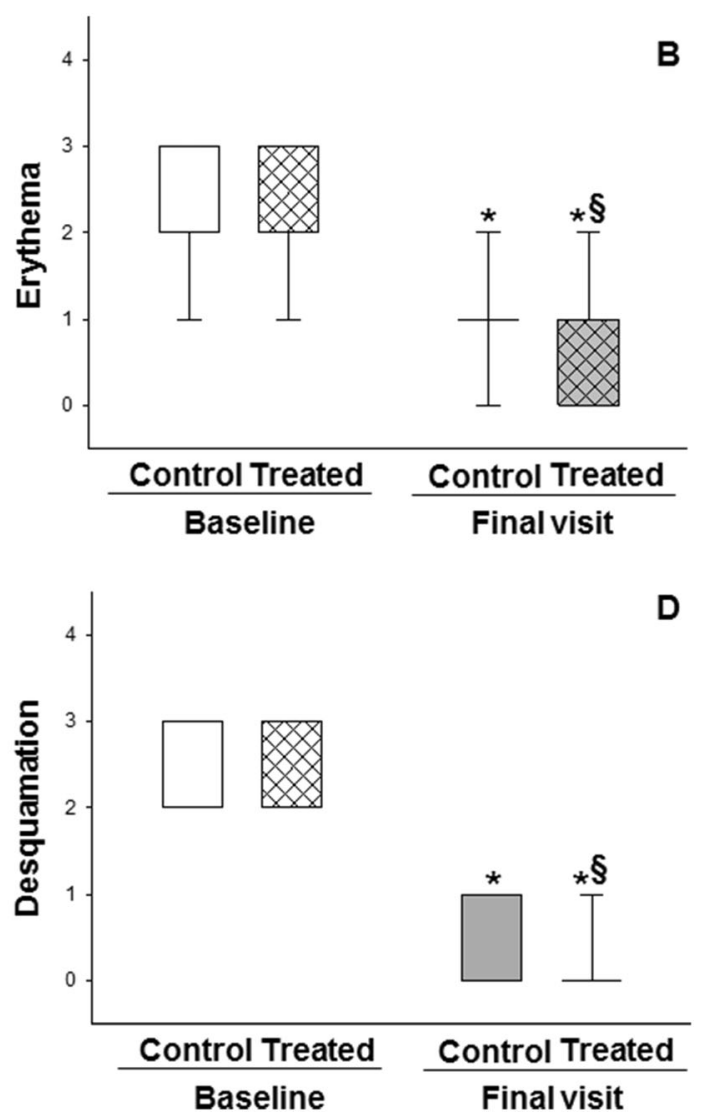

treated and control sides, $\mathbf{b}$ changes in erythema after exposure to NB-UVB therapy with or without UV Fotogel treatment, c effects of the phototherapy and the UV Fotogel on infiltration, $\mathbf{d}$ values of desquamation of skin with and without UV Fotogel application. See section "LPSI, Cosmetic Acceptability, and Tolerability” for description of scores

control side (0.0 [25p-75p: $0.0-0.0]$ vs. 0.0 [0.0-1.0], respectively) (Fig. 3d).

\section{Application of UV Fotogel With NB-UVB Was Not Accompanied By Considerable Adverse Effects and the Preparation Was Found to be Cosmetically Acceptable}

At the final visit, patients were asked to report any adverse effects of using UV Fotogel. Two patients reported a mild burning sensation, three patients experienced mild itching, and two patients experienced moderate itching. Two patients reported mild stretching. None of 
the patients reported stabbing pain and tingling. No severe symptoms were reported.

The majority of the patients (21 patients) found the cosmetic acceptability of UV Fotogel excellent, with ratings of very good and good given by 11 and 11 patients, respectively. Only one patient found the cosmetic acceptability sufficient (Table 1).

\section{Toleration to and Cosmetic Acceptability of Applying UV Fotogel Prior to Sunbathing}

The use of UV Fotogel prior to sunbathing (Part II of the study) led to a considerable decrease in median L-PSI score, was well tolerated by the patients, and the cosmetic acceptability was assessed to be good.

The median L-PSI score of patients in Part II at baseline (6.0 [25p-75p: 6.0-7.0]) had significantly decreased by the final visit $(2.5[25 \mathrm{p}-75 \mathrm{p}$ :
1.5-3.5]). At the final visit, one patient reported mild burning sensations and two patients reported moderate burning sensations. One patient experienced mild stabbing pain and another experienced moderate stabbing pain. Mild itch was reported by four patients and moderate itch by three patients. One patient felt mild stretching of the skin, and another patient reported moderate stretching of the skin. No patients reported tingling sensations. Six patients considered the cosmetic acceptability to be excellent, 15 patients found it to be very good, 18 patients evaluated it as good, and six patients found it to be sufficient (Table 2).

\section{DISCUSSION}

The treatment of psoriasis depends on the severity of the disease. In mild to moderate

Table 1 Tolerability, irritability, and cosmetic acceptability of UV Fotogel application in combination with narrow bandultraviolet B phototherapy

Tolerability and irritability of UV Fotogel with NB-UVB phototherapy

\begin{tabular}{llllll}
\hline Degree of the symptom $^{\mathbf{a}}$ & \multicolumn{2}{l}{ Symptoms } & & \\
\cline { 2 - 6 } & Burning & Stabbing pain & Itching & Stretching & Tingling \\
\hline 1 & $42(95.5 \%)$ & $44(100 \%)$ & $39(88.6 \%)$ & $42(95.5 \%)$ & $44(100 \%)$ \\
2 & $2(4.5 \%)$ & $0(0 \%)$ & $3(6.8 \%)$ & $2(4.5 \%)$ & $0(0 \%)$ \\
3 & $0(0 \%)$ & $0(0 \%)$ & $0(0 \%)$ & $0(0 \%)$ & $0(0 \%)$ \\
\hline
\end{tabular}

Cosmetic acceptability

\begin{tabular}{lc}
\hline Score $^{\mathbf{b}}$ & $\boldsymbol{n}(\%)$ \\
\hline 1 & $0(0 \%)$ \\
2 & $1(2.3 \%)$ \\
3 & $11(25 \%)$ \\
4 & $11(25 \%)$ \\
5 & $21(47.7 \%)$
\end{tabular}

Values in table are presented as the number of patients, with the percentage of the reporting population given in parenthesis $N B-U V B$ Narrow band-ultraviolet $\mathrm{B}$

a 1 , The given symptom is not present; 2 , mild symptoms; 3 , moderate symptoms; 4 , severe symptoms. Note: no patient reported moderate or severe symptoms

b 1, Poor acceptability; 2, sufficient; 3, good; 4, very good; 5, excellent 
Table 2 Tolerability, irritability, and cosmetic acceptability after application of UV Fotogel with sunbathing

Tolerability and irritability of UV Fotogel with sunbathing

\begin{tabular}{|c|c|c|c|c|c|}
\hline \multirow[t]{2}{*}{ Degree of the symptom ${ }^{a}$} & \multicolumn{5}{|l|}{ Symptoms } \\
\hline & Burning & Stabbing pain & Itching & Stretching & Tingling \\
\hline 1 & $42(93.3 \%)$ & $43(95.6 \%)$ & $38(84.4 \%)$ & $43(95.6 \%)$ & $45(100 \%)$ \\
\hline 2 & $1(2.2 \%)$ & $1(2.2 \%)$ & $4(8.9 \%)$ & $1(2.2 \%)$ & $0(0 \%)$ \\
\hline 3 & $2(4.4 \%)$ & $1(2.2 \%)$ & $3(6.7 \%)$ & $1(2.2 \%)$ & $0(0 \%)$ \\
\hline \multicolumn{6}{|l|}{ Cosmetic acceptability } \\
\hline Score $^{b}$ & & & & & $n(\%)$ \\
\hline 1 & & & & & $0(0 \%)$ \\
\hline 2 & & & & & $6(13.3 \%)$ \\
\hline 3 & & & & & $18(40 \%)$ \\
\hline 4 & & & & & $15(33.3 \%)$ \\
\hline 5 & & & & & $6(13.3 \%)$ \\
\hline
\end{tabular}

Values in table are presented as the number of patients, with the percentage of the reporting population given in parenthesis

a 1 , The given symptom is not present; 2 , mild symptoms; 3 , moderate symptoms; 4 , severe symptoms. Note: no patient reported moderate or severe symptoms

b 1, Poor acceptability; 2, sufficient; 3, good; 4, very good; 5, excellent

psoriasis, topical therapy is indicated alone or in combination with UV phototherapy.

NB-UVB is currently the first-line phototherapy for stable plaque psoriasis. Compared to broadband UVB phototherapy, NB-UVB results in a faster reduction of lesions, with longer remissions expected [10]. Nevertheless, careful design of the therapy is necessary, as UV light might lead to cataracts, photodermatitis, and photoaging. Carcinogenesis is also a potential risk [18]. However, the efficacy of UVB therapy can potentially be increased by combining it with different topical and systemic agents. Historically, UVB phototherapy was combined with coal tar; however, this preparation smells, stains skin, has irritative and carcinogenic properties, and may block UVB rays. Dithranol has also applied to the skin to improve the efficacy of UVB therapy, but this preparation is associated with an increased risk of irritation [19]. Other topical treatments used just before UV irradiation (e.g., different emollients, ichthyol, and calcipotriol) block the penetration of UV light, thereby decreasing the therapeutical efficacy of the phototherapy [20-22]. In the present study, we combined NBUVB with a preparation which, when used alone, does not have a therapeutic effect, but which may enhance the efficacy of phototherapy.

The optical properties of the psoriatic skin differ from those of normal skin [17, 23]. The multiple air-keratin interfaces in psoriatic scales allow reflection and refraction of incident radiation, and the lesions, thereby, act as a partial optic barrier. Successful treatment of psoriasis with UV-light requires adequate penetration of the radiation to at least the level of viable epidermal cells.

Compared to other combination therapies, there appear to be fewer possibilities to modify the transmission of UVB. A saltwater shower and application of lipophilic compounds may be beneficial $[19,24]$. These latter compounds reduce the fraction of incident radiation which is then backscattered or re-emitted from the 
skin. It has been confirmed in vivo that the application of non-photosensitizing oily lubricants to the skin increases the transmission of light through psoriatic plaques, resulting in increased effectivity of the phototherapy $[15,17,23]$. Mineral oil (e.g., liquid paraffin) can penetrate and fill the air-stratum corneum interfaces, thereby facilitating the transmission of radiation. It is currently the only Medicareapproved treatment to increase the efficacy of UVB phototherapy in the USA. However, the majority of psoriasis treating centers have reported that they do not routinely use emollients before UV irradiation [16].

The lack of randomized controlled studies on the benefit of using emollients concomitantly with UVB is a further difficulty. The only clinical investigation performed to date was conducted with paraffin oil in children. This study reported a significantly greater improvement in scaling, induration, and area of involvement after application of mineral oil while no adverse effects were observed [16]. Nevertheless, mineral oil is considered to be cosmetically unfavorable.

Although mineral oil stains clothes and patients encounter difficulties in dressing after the treatment, UVB with mineral oil is the most frequently used phototherapy for psoriasis in the USA according to an analysis of Healthcare Common Procedure Coding System (HCPCS) codes. In 2015, approximately 400,000 UVB therapy treatments with mineral oil (HCPCS code 96910) were performed in the USA, and the corresponding USD 21.7 million cost accounted for $48 \%$ of the total Medicare budget for phototherapy [25]. Other emollient preparations (e.g., tar, salicylic acid, thickly applied petrolatum) are cosmetically more favorable, but have the disadvantages of absorbing UV light, increasing MED, and slowing the clearance of psoriasis [15, 26, 27].

The components of the UV Fotogel were selected solely on their optical properties, as the aim was to develop a medical device that decreases the reflection of UVB light while at the same time blocks the shorter wavelengths. The absorption spectra of different compounds and the UV transmittance through the gel were measured in vitro [28]. Based on the results of our previous in vitro examination, the components of UV Fotogel facilitate the penetration of UVB wavelengths in the 310- to 320-nm range into the skin and block wavelengths in the range of $280-300 \mathrm{~nm}$ [28]. Thus, the preparation facilitates penetration of the therapeutically useful fraction and blocks the range which may evoke an inflammatory reaction (Fig. 4). Moreover, topical use of the listed components can be considered safe [29-31]. The findings of the present study confirm the beneficial effect of the preparation. After exposure to NB-UVB, L-PSI values were found to be significantly lower in UV Fotogel-treated lesions than in skin not treated with UV Fotogel. Moreover, each parameter of the L-PSI (erythema, infiltration, and desquamation) showed a considerable improvement with the application of UV Fotogel compared to the control skin. In addition to the beneficial therapeutic effect, the preparation was well tolerated, and the cosmetic acceptability was good.

Further interesting results were provided by Part II, in which UV Fotogel was used by patients prior to sunbathing. Although sunbathing is not considered to be a first-line therapy in psoriasis [10], UV radiation from the sun is an effective and natural treatment for the disease [32]. The results from this part of the study confirm that the preparation is cosmetically favorable and free of considerable adverse reactions under the circumstances of home application.

The limitations of our study include the relatively low number of enrolled patients in Part I and the lack of dosimetry in Part II.

Regarding the applicability of the results in the general population, worldwide approximately 200 million patients have only mild psoriasis that is frequently treated by natural sunlight. However, no approved medical device is as yet available to increase the transmittance of UVB light by reducing the reflectance of the sunlight. Since UV Fotogel allows and enhances the penetration of therapeutically useful wavelengths (310-320 $\mathrm{nm}$ ) and blocks the most erythematogenic part of the UVB spectrum below $300 \mathrm{~nm}$, this medical device may improve the therapeutic effects of natural sunlight in millions of affected individuals. 

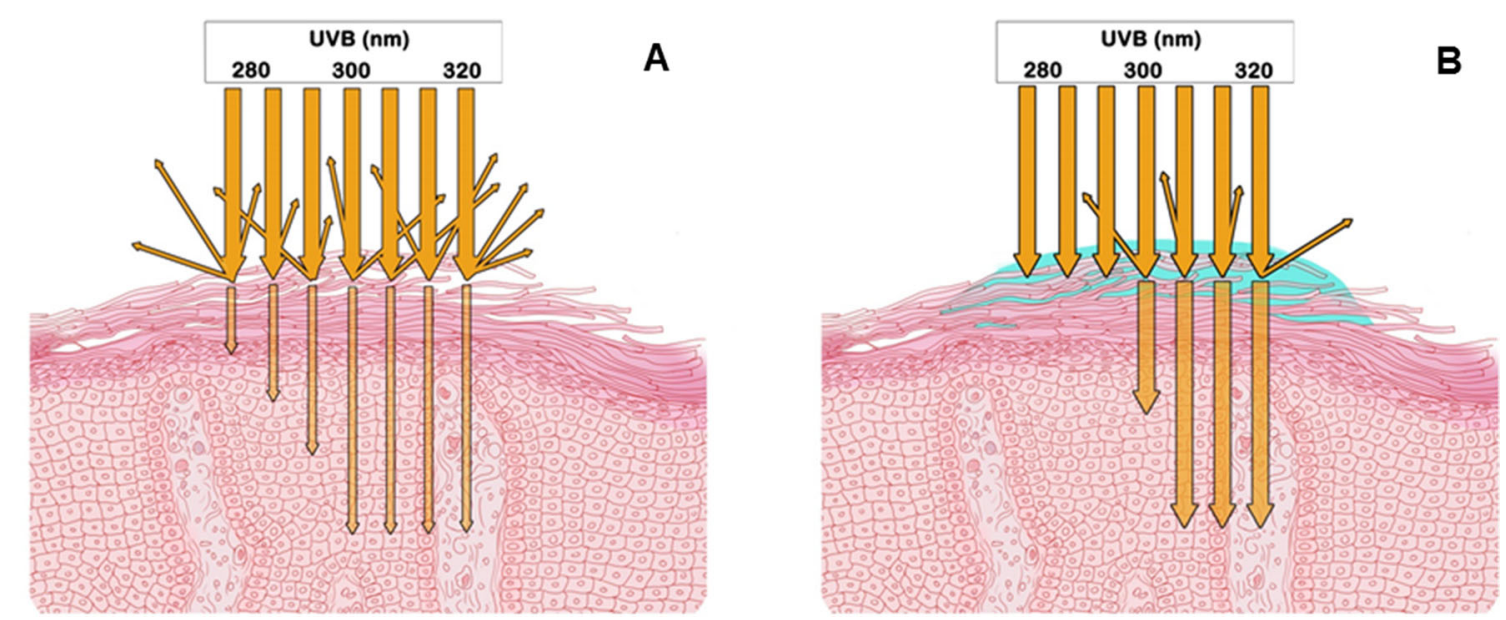

Fig. 4 Penetration of UVB radiation into the skin without (a) and with the use of UV Fotogel (b)

Although the preparation has been primarily designed for the treatment of psoriasis, the treatment of other diseases that are responsive to UV therapy (e.g., atopic dermatitis, earlystage cutaneous T-cell lymphoma) might possibly also be enhanced with UV Fotogel. However, further investigation is necessary to determine this.

\section{CONCLUSIONS}

In conclusion, application of a novel preparation containing sodium hyaluronate and nicotinic acid prior to NB-UVB phototherapy led to a greater reduction of psoriatic lesions than NBUVB phototherapy alone. Furthermore, the cosmetic acceptability of the preparation was good, and there were no reports of considerable side effects. The preparation was also well tolerated by patients who used it prior to sunbathing. The results suggest that UV Fotogel might be a useful device for the enhancement of phototherapy in psoriasis.

\section{ACKNOWLEDGEMENTS}

We thank the participants of the study.

Funding. The journals rapid Service Fee is funded by the research Grant EFOP-3.6.2-16-
2017-00009 provided by the European Union and the Hungarian Government and by the EU's H2020 Grant Agreement No. 739593.

Authorship. All named authors meet the International Committee of Medical Journal Editors (ICMJE) criteria for authorship for this article, take responsibility for the integrity of the work as a whole, and have given their approval for this version to be published.

Authorship Contributions. Lajos Kemény and Eszter Baltás are shared last authors.

Disclosures. Lajos Kemény has an approved patent on the components of UV Fotogel ${ }$. Balázs Bende, Róbert Kui, Annamária Németh, Mariann Borsos, Zoltán Tóbiás, Gábor Erős and Eszter Baltás have nothing to disclose.

Compliance with Ethics Guidelines. The study was approved by the National Institute of Pharmacy and Nutrition (Hungary) (permission numbers: OGYÉI/723/2017 and OGYÉI/28552/ 2017) and was performed in accordance with the Helsinki Declaration of 1964 and its later amendments. Informed consent was obtained from all participants.

Data Availability. The datasets generated and analyzed during the current study are available from the corresponding author on reasonable request. 
Open Access. This article is licensed under a Creative Commons Attribution-NonCommercial 4.0 International License, which permits any non-commercial use, sharing, adaptation, distribution and reproduction in any medium or format, as long as you give appropriate credit to the original author(s) and the source, provide a link to the Creative Commons licence, and indicate if changes were made. The images or other third party material in this article are included in the article's Creative Commons licence, unless indicated otherwise in a credit line to the material. If material is not included in the article's Creative Commons licence and your intended use is not permitted by statutory regulation or exceeds the permitted use, you will need to obtain permission directly from the copyright holder. To view a copy of this licence, visit http:// creativecommons.org/licenses/by-nc/4.0/.

\section{REFERENCES}

1. Parisi R, Symmons DP, Griffiths CE, Aschroft DM. Global epidemiology of psoriasis: a systemic review of incidence and prevalence. J Invest Dermatol. 2013;133:377-85.

2. Kim WB, Jerome D, Yeung J. Diagnosis and management of psoriasis. Can Fam Phys. 2017;63: 278-85.

3. Feldman SR, Fleischer AB Jr, Cooper JZ. New topical treatments change the pattern of treatment of psoriasis: dermatologists remain the primary providers of this care. Int J Dermatol. 2000;39:41-4.

4. Horn EJ, Fox KM, Patel V, Chiou CF, Dann F, Lebwohl M. Are patients with psoriasis undertreated? Results of National Psoriasis Foundation survey. J Am Acad Dermatol. 2007;57:957-62.

5. Menter A, Korman NJ, Elmets CA, et al. Guidelines of care for the management of psoriasis and psoriatic arthritis: Section 3. Guidelines of care for the management and treatment of psoriasis with topical therapies. J Am Acad Dermatol. 2009;60:643-59.

6. Ashcroft DM, Po AL, Williams HC, Griffith CE. Systematic review of comparative efficacy and tolerability of calcipotriol in treating chronic plaque psoriasis. BMJ. 2000;320:963-7.
7. Mason AR, Mason J, Cork M, Dooley G, Edwards G. Topical treatments for chronic plaque psoriasis. Cochrane Database Syst Rev. 2009;(2): CD005028.

8. Goldenberg G, Lanoue J, Dong J. New oral therapies for psoriasis: a comprehensive review. J Clin Aesthet Dermatol. 2016;9:25-8.

9. Lin VW, Ringold S, Devine EB. Comparison of ustekinumab with other biological agents for the treatment of moderate to severe plaque psoriasis: a Bayesian network meta-analysis. Arch Dermatol. 2012;148:1403-10.

10. Menter A, Gottlieb A, Feldman SR, et al. Guidelines of care for the management of psoriasis and psoriatic arthritis: Section 1. Overview of psoriasis and guidelines of care for the treatment of psoriasis with biologics. J Am Acad Dermatol. 2008;58:826-50.

11. Zhang $\mathrm{P}, \mathrm{Wu} \mathrm{MX}$. A clinical review of phototherapy for psoriasis. Lasers Med Sci. 2018;33:173-80.

12. Bónis B, Kemény L, Dobozy A, Bor Z, Szabó G, Ignácz F. 308 nm UVB excimer laser for psoriasis. Lancet. 1997;350:1522.

13. Kemény L, Bónis B, Dobozy A, Bor Z, Szabó G, Ignácz F. 308-nm excimer laser therapy for psoriasis. Arch Dermatol. 2001;137:95-6.

14. Novák Z, Bónis B, Baltás E, et al. Xenon chloride ultraviolet $\mathrm{B}$ laser is more effective in treating psoriasis and in inducing $\mathrm{T}$ cell apoptosis than narrowband ultraviolet B. J Photochem Photobiol B. 2002;67:32-8.

15. Fetil E, Akarsu S, Ilknur T, Kusku E, Günes AT. Effects of some emollients on the transmission of ultraviolet. Photodermatol Photoimmunol Photomed. 2006;22:137-40.

16. Otman SG, Edwards C, Pearse AD, Gambles BJ, Anstey AV. Modulation of ultraviolet (UV) transmission by emollients: relevance to narrowband UVB phototherapy and psoralen plus UVA photochemotherapy. Br J Dermatol. 2006;154:963-8.

17. Jain VK, Bansal A, Aggarwal K, Jain K. Enhanced response of childhood psoriasis to narrow-band UVB phototherapy with preirradiation use of mineral oil. Pediatr Dermatol. 2008;25:559-64.

18. Seebode C, Lehmann J, Emmert S. Photocarcinogenesis and skin cancer prevention strategies. Anticancer Res. 2016;36:1371-8.

19. Benáková N. Phototherapy of psoriasis in the era of biologics: still in. Acta Dermatovenerol Croat. 2011;19:195-205. 
20. Mrowietz U, Kragballe K, Reich K, et al. Definition of treatment goals for moderate to severe psoriasis: a European consensus. Arch Dermatol Res. 2011;303:1-10.

21. Samarasekera E, Sawyer L, Parnham J, Smith CH. Assessment and management of psoriasis: summary of NICE guidance. BMJ. 2012;345:e6712.

22. Warnecke J, Wendt A. Antiinflammatory action of pale sulfonated shale oil in UVB erythema test. Inflamm Res. 1998;47:75-8.

23. Anderson RR, Parrish JA. The optics of human skin. J Invest Dermatol. 1981;77:13-9.

24. Gambichler T. Balneophototherapy for psoriasis saltwater baths and UV-B irradiation, revisited. Arch Dermatol. 2007;143:647-9.

25. Tan SY, Buzney E, Mostaghimi A. Trends in phototherapy utilization among Medicare beneficiaries in the United States, 2000 to 2015. J Am Acad Dermatol. 2018;79:672-9.

26. Hudson-Peacock MJ, Diffey BL, Farr PM. Photoprotective action of emollients in ultraviolet therapy of psoriasis. Br J Dermatol. 1994;130:361-5.
27. Lebwohl M, Martinez J, Weber P, DeLuca R. Effects of topical preparations on the erythemogenicity of UVB: implications for psoriasis phototherapy. J Am Acad Dermatol. 1995;32:469-71.

28. Kemény L, Révész $P$, Csányi E. Composition for increasing of the effectiveness of UV-B therapy, process for the preparation thereof, and its use. Patent No.: US9839595. Alexandria: US Patent and Trademark Office.

29. Jang HJ, Shin CY, Kim KB. Safety evaluation of polyethylene glycol (PEG) compounds for cosmetic use. Toxicol Res. 2015;31:105-36.

30. DRUGBANK. https://www.drugbank.ca/drugs/ DB00627.

31. Becker LC, Bergfeld WF, Belsito DV, et al. Final report of the safety assessment of hyaluronic acid, potassium hyaluronate, and sodium hyaluronate. Int J Toxicol. 2009;28:5-67.

32. Nilsen LT, Soyland E, Krogstad AL. Estimated ultraviolet doses to psoriasis patients during climate therapy. Photodermatol Photoimmunol Photomed. 2009;25:202-8. 